The Journal of

Obstetrics and

Gynaecology

\section{Journal of Obstetrics and Gynaecology}

ISSN: 0144-3615 (Print) 1364-6893 (Online) Journal homepage: http://www.tandfonline.com/loi/ijog20

\title{
Computerised analysis of antepartum foetal heart parameters: New reference ranges
}

\section{Natascia Giuliano, Maria Laura Annunziata, Francesca Giovanna Esposito, Salvatore Tagliaferri, Andrea Di Lieto, Giovanni Magenes, Maria Gabriella Signorini, Marta Campanile \& Domenico Arduini}

To cite this article: Natascia Giuliano, Maria Laura Annunziata, Francesca Giovanna Esposito, Salvatore Tagliaferri, Andrea Di Lieto, Giovanni Magenes, Maria Gabriella Signorini, Marta Campanile \& Domenico Arduini (2016): Computerised analysis of antepartum foetal heart parameters: New reference ranges, Journal of Obstetrics and Gynaecology

To link to this article: http://dx.doi.org/10.1080/01443615.2016.1239069

\section{曲 Published online: 06 Dec 2016.}

\section{Submit your article to this journal ๘}

Џ Article views: 11

Q View related articles $\asymp$

View Crossmark data $־$ 


\title{
Computerised analysis of antepartum foetal heart parameters:
} New reference ranges

\author{
Natascia Giuliano ${ }^{a}$, Maria Laura Annunziata ${ }^{a}$, Francesca Giovanna Esposito ${ }^{a}$, Salvatore Tagliaferri ${ }^{a}$ (D), \\ Andrea Di Lieto ${ }^{a}$, Giovanni Magenes ${ }^{b}$, Maria Gabriella Signorini ${ }^{c}$, Marta Campanile ${ }^{a}$ and Domenico Arduini ${ }^{d}$ \\ ${ }^{\mathrm{a} D e p a r t m e n t}$ of Obstetrical-Gynaecological, Urological Science and Reproductive Medicine, Federico II University, Naples, Italy; ${ }^{\mathrm{b}}$ Department \\ of Electrical, Computer and Biomedical Engineering, University of Pavia, Pavia, Italy; 'Department of Electronic, Information and \\ Bioengineering, Politecnico of Milano, Milan, Italy; ${ }^{\mathrm{d}}$ Department of Obstetrics and Gynaecology, Foetal Medicine Centre, University of Rome \\ "Tor Vergata", Rome, Italy
}

\section{ABSTRACT}

We selected 4012 cCTG records (one trace for each patient) performed in healthy pregnancies from 30th to 42nd gestational week using foetal heart rate (FHR), short-term variability (STV), long-term irregularity (LTI), Delta, approximate entropy (ApEn), spectral components as low frequency (LF), median frequency (MF), high frequency (HF) and LF/(HF + MF) ratio were analysed. Reference nomograms were created and sensitivity and specificity for the prediction of foetal compromise were calculated which were $90 \%$ and $89 \%$, respectively. Changes of cCTG parameters according to gestational week were evaluated: FHR $(r=-.65)$ and LF $(r=-.87)$ showed a statistically significant reduction $(p<.05)$ with gestational age. STV $(r=.59)$, LTI $(r=.69)$, Delta $(r=.67)$, and MF $(r=.88)$ showed a statistically significant increase $(p<.05)$ with gestational age. In contrast, for ApEn $(r=-.098)$, HF $(r=.14)$ and LF/(HF + MF) ratio $(r=-.47)$ a non-statistically significant change was found $(p>.05)$. The identification of reference ranges for CCTG indexes in according to gestational age could provide a more objective examination of cCTG trace.

\section{KEYWORDS}

Computerised cardiotocography; antepartum foetal monitoring; foetal heart rate; gestational age; reference ranges

\section{Introduction}

Cardiotocography is the most widespread method of foetal surveillance worldwide. During the last decades, traditional monitoring systems received a fundamental improvement by new technological devices allowing the overcoming of subjective interpretation while providing objective information about foetal wellbeing (Bernardes et al. 1997; Figueras et al. 2005).

Computerised cardiotocography (cCTG) improves foetal monitoring reliability as it standardises CTG evaluation with automatic estimation of foetal heart rate (FHR) parameters (Signorini et al. 2003). In fact, cCTG is characterised by objectivity and consistency (Dawes et al. 1996) as it performs an automatic trace analysis, implementing diagnostic criteria accepted in clinical obstetric practise (Gagnon et al. 1993; Pardey et al. 2002).

Nowadays, the available foetal monitoring guidelines, published by the American College of Obstetricians and Gynaecologists (ACOG) (ACOG 1989, 1995, 2005, 2009), the National Institute of Child Health and Human Development (NICHHD) (Zuspan et al. 1979; NICHHD 1997; Macones et al. 2008), the Royal College of Obstetricians and Gynaecologists (RCOG) (RCOG 2001), and the National Institute of Clinical Excellence (NICE) (NICE 2007) focus specifically on intrapartum monitoring, although some try to apply the same principles to antepartum tracings (Macones et al. 2008; ACOG 2009). The use of computerised analysis during the antepartum period has some problems. The first one is the difficulty of transforming the linguistic description of FHR parameters into numerical algorithms (Wróbel et al. 2013). Second, even when various algorithms have been developed, only some CCTG parameters have been investigated. In fact, one of the most widespread system of analysis, the Oxford Foetal Care System (Serra et al. 2009) analyses only linear parameters such as baseline foetal heart rate (Basal FHR), long-term FHR variation (LTV), short-term FHR variation (STV), episodes of high/low FHR variation, accelerations and decelerations. While many studies were based on the Oxford analyses, few studies focussed their attention on CCTG trace analysis using the 2CTG2 system that measures additional CCTG parameters such as non-linear features. Moreover, the major limitations are related to the few attempts to evaluate clinical implication of cCTG parameters as the absence of their analysis in relation with neonatal outcome. Most of the studies focussed on the predictive value of CCTG parameters considered only their relation with the Apgar score, not including other important parameters such as umbilical cord $\mathrm{pH}$ at birth (Bernardes et al. 1997; Park et al. 2001).

CONTACT Natascia Giuliano natascia.giuliano@hotmail.it $=$ Department of Obstetrical-Gynaecological, Urological Science and Reproductive Medicine of the Federico II University, Naples, Italy 
In the present study we investigated several aspects of cCTG analysis trying to simplify its clinical application.

The aims of our study were:

1. Evaluating how the CCTG (linear and non-linear) parameters analysed with the 2CTG2 system change through gestation. This could provide new information about the clinical meaning of numerical parameters, and they, in turn, may explain physiological or paraphysiological phenomena that occur in foetus.

2. Creating reference normality values for all CCTG parameters investigated, as a function of gestational age, in a population of healthy pregnancies. Supplementing the first tables developed by Arduini et al. (1993), we wanted to provide new reference values for each week, from the 30th to the 42th week, for uncomplicated pregnancies, based on our clinical experience and using our system.

3. Providing a clinical validation of the 2CTG2 system to evaluate the usefulness of our system and the new tables, in relationship with foetal outcome.

\section{Materials and methods}

\section{Data collection}

This is a prospective study performed from October 2012 to October 2014. The population study consists of pregnant women who underwent to antenatal computerised cardiotocographic exam at the Department of ObstetricalGynaecological and Urological Science and Reproductive Medicine of the Federico II University, Naples, Italy. The principles of the Helsinki Declaration were followed, and a written consent authorised the use of clinical information from cCTG records of each patient for scientific aims. We used Philips Avalon Foetal Monitor FM30 cardiotocographs, with an ultrasound transducer and a transabdominal tocodynamometer. The Cardiotocograph was interfaced to 2CTG2 system (SEA, Italy) for computerised analysis (Arduini et al. 1993). We used Mantel's algorithm, based on a low-pass digital system that crosses the tracing five times, starting from a value determined by histogram analysis of the FHR distribution (Mantel et al. 1990). In fact, Mantel's algorithm is generally satisfactory when the FHR tracings are regular with long and stable FHR segments, which are found most commonly during the antepartum period (Nidhal et al. 2011).

From 9732 cCTG records archived during these years, we selected 4012 antenatal nonstress CCTG traces from 30th to 42nd gestational week, associated with documented maternal and foetal normal outcome. cCTG traces were carried out for routine antenatal care ("term foetuses") or as control ("preterm feuses", for the study aim). Only one trace per foetus was analysed and the selected CCTG trace was the first performed at our Department.

Gestational age was obtained from foetal crown-rump length ultrasonographic measurement performed at 8-14 weeks. The last menstrual period-based date was only corrected if the interval between the ultrasound and the menstrual dates was greater than 1 week (Butt et al. 2014).

Inclusion criteria were: Caucasian ethnic, singleton healthy pregnancies, neonatal weight above the 10th percentile for gestational age and sex (Hadlock et al. 1985), absence of structural or chromosomal abnormalities, normal umbilical artery Doppler (Yeh et al. 2012), absence of maternal diseases. Traces from foetuses below the 30th gestational week were excluded because the most part of them did not meet the inclusion criteria.

FHR signals were obtained in a controlled clinical environment, with the mothers lying in a semi-Fowler's position in a relaxed condition. Each nonstress test was at least 20 minutes long. The FHR analysis was carried out in each signal using a 10-min segment of trace in order to obtain the same length of analysis segment. We selected segment in absence of large FHR alterations (e.g. accelerations and/or decelerations) and other phenomena such us uterine contraction and/or foetal movements. Only records of acceptable quality (signal loss $<50 \%$ ) were included in the study.

The parameters assessed are divided in: time domain parameters (baseline FHR, STV, LTI, Delta), frequency domain parameters, computed by adopting a power spectral estimation (LF, MF, HF and LF/(HF + MF) ratio) and nonlinear parameter (ApEn).The resulting $c C T G$ indexes were computed for each gestational week. The CCTG records were divided by gestational age: 30 th week $\left(n_{\text {rec }}=157\right), 31$ st week $\left(n_{\text {rec }}=169\right)$, 32 nd week $\left(n_{\text {rec }}=210\right), 33$ rd week $\left(n_{\text {rec }}=217\right), 34$ th week $\left(n_{\text {rec }}=249\right), 35$ th week $\left(n_{\text {rec }}=237\right), 36$ th week $\left(n_{\text {rec }}=378\right)$, 37 th week $\left(n_{\text {rec }}=456\right)$, 38th week $\left(n_{\text {rec }}=425\right)$, 39th week $\left(n_{\text {rec }}=516\right)$, 40th week $\left(n_{\text {rec }}=432\right), 41$ st week $\left(n_{\text {rec }}=369\right)$, 42nd week ( $\left.n_{\text {rec }}=197\right)$.

\section{Acquisition and elaboration of CTG signals}

FHR signals were acquired at a sampling frequency of $2 \mathrm{~Hz}$. If the signals are measured in beats per minute (bpm), we called them $\mathrm{S}_{120}(\mathrm{i})$, where 120 is the number of samples in a minute (i); if they are expressed in milliseconds, we called them $\mathrm{T}_{120}(\mathrm{i})$. For the computation of FHR parameters we used a signal obtained by opportunely under sampling $S_{120}(i)$. This is accomplished by replacing each group of five consecutive points in the original series with their average value; in this way the resulting signal is composed of 24 points per minute $(0.4 \mathrm{~Hz})$. We referred to this signal as $\mathrm{S}_{24}(\mathrm{i})$ if the signal is expressed in bpm, or as $\mathrm{T}_{24}(\mathrm{i})$ if it is expressed in milliseconds. The 2CTG2 programme always computes the parameters on the entire signal. During the acquisition, all the parameters are updated every minute.

\section{Baseline, accelerations and decelerations}

The baseline is a running average of the FHR where accelerations and decelerations are defined as deviation of the FHR from the baseline lasting a sufficient amount of time. In the 2CTG2 software, the baseline is computed by using the algorithm developed by Mantel (Mantel et al. 1990; Preboth 2000). Accelerations and decelerations are defined as follows (Arduini et al. 1993):

- Small acceleration: FHR elevation over the greater baseline between 10 and $15 \mathrm{bpm}$ for at least $15 \mathrm{~s}$.

- Big acceleration: FHR elevation over the greater baseline of $15 \mathrm{bpm}$ for at least $15 \mathrm{~s}$. 
- Decelerations:

- FHR lower than the baseline for an amplitude of at least $20 \mathrm{bpm}$ and for a duration of at least $30 \mathrm{~s}$.

- FHR lower than the baseline for an amplitude of at least $10 \mathrm{bpm}$ and for a duration of at least $60 \mathrm{~s}$.

\section{Short-term variability}

STV quantifies FHR variability over a very short time scale, usually on a beat to beat basis. In the automated system 2CTG2, this index is implemented by adopting the definition of Arduini et al. (1993). Given one minute of RR signal, $T_{24}(\mathrm{i})$ in $\mathrm{ms}$ and $\mathrm{i} \in[1 ; 24]$, STV is defined as

$$
S T V=\text { mean }\left[\left|T_{24}(i+1)-T_{24}(i)\right|\right]_{i}=\frac{\sum_{i=1}^{23}\left|T_{24}(i+1)-T_{24}(i)\right|}{23}
$$

Arduini excludes from the calculation big accelerations and decelerations.

\section{Long-term irregularity}

Given a three minutes $T_{24}(i)$ signal with $i \in[1 ; 72]$, LTI is defined as the interquartile range $(1 / 4 ; 3 / 4)$ of the distribution of the modula $m_{24}(j)$ with $i \in[1 ; 71]$

$$
m_{24}(j)=\left(T_{24}^{2}(j+1)+T_{24}^{2}(j)\right)^{1 / 2}
$$

The definition is the same provided by De Haan (ACOG 1989), with the exception of a window of 72 (and not 512) samples long.

Arduini excludes from the calculation big accelerations and decelerations.

\section{Delta}

Given a minute of signal in millisecond $T_{24}(i)$ with $i \in[1 ; 24]$, Delta is defined as the difference between the maximum and minimum FHR value,

$$
\text { Delta }=\operatorname{maxT}_{24}(\mathrm{i})-\operatorname{minT}_{24}(\mathrm{i})
$$

Arduini et al. (1993) excludes from the calculation big accelerations and decelerations.

\section{Approximate entropy}

ApEn is a collection of statistical indexes. It measures the regularity and, indirectly, the correlation and the persistence of a signal: small values indicate reduced signal irregularity. We use the original definition by Pincus (1995):

$$
\operatorname{ApEn}_{-}(m, r)=\frac{\sum_{\mathrm{i}=1}^{N-m+1} \log C_{\mathrm{i}}(m, r)}{N-m+1}-\frac{\sum_{\mathrm{i}=1}^{N-m} \log C_{\mathrm{i}}(m+1, r)}{N-m}
$$

where $m$ is a natural number, $r$ a positive real and $N=360$. The approximate entropy is computed over windows of FHR signal 3 min long.

\section{Spectral analysis}

Parameters in the frequency domain were obtained from autoregressive power spectrum estimation. In the FHR spectrum we identify four contributions: the very low frequency (VLF $0-0.03 \mathrm{~Hz}$ ), which is related to long period and non-linear contributions, the low frequency (LF $0.03-0.15 \mathrm{~Hz}$ ), which is mainly correlated with neural sympathetic activity and the high frequency (HF $0.5-1 \mathrm{~Hz}$ ), which marks the presence of foetal breathing. The middle frequency (MF $0.15-0.5 \mathrm{~Hz}$ ) depends on foetal movements and maternal breathing activity and it is typical of FHR spectrum. LF/ $(\mathrm{HF}+\mathrm{MF})$ ratio quantifies the autonomic balance between neural control mechanisms from different origin (Signorini et al. 2003).

\section{Statistical analysis}

The statistical software package IBM SPSS 20.0 for Windows was used for data analysis. FHR, STV, LTI, Delta, ApEn and spectral components, as LF, MF, HF, LF/(HF + MF) ratio and their distribution throughout third trimester of gestation were analysed. The Kolgomorov-Smirnov test for normality showed a non-normal distribution. The Kruskal-Wallis test was computed to evaluate the statistical significant variation of CCTG parameters with gestational age. The relationship between CCTG parameters and gestational age was analysed through the Spearman's rank correlation coefficient. Threshold for statistical significance was $p<.05$.

The following percentiles for each parameter were calculated: 5th; 50th; 95th.

In order to verify the clinical validity of our reference ranges, we realised a second study that included

A new sample of newborns made up 328 babies: 118 with a favourable outcome $(\mathrm{pH}>7.05$ and $\mathrm{BD}<12 \mathrm{mmol} / \mathrm{L}$, normal Apgar scores $>7$ both at 1 and $5 \mathrm{~min}$, delivery at/or beyond 37th gestational week, intact survival beyond 28 days of postnatal life), 210 with a unfavourable outcome $(\mathrm{pH}$ $<7.05$ and $\mathrm{BD}>12 \mathrm{mmol} / \mathrm{L}$, normal Apgar scores $<7$ both at 1 and $5 \mathrm{~min}$, delivery before 37th gestational week, necessity of "neonatal intensive care unit for $24 \mathrm{~h}$ "). Antepartum cCTG traces performed by their mothers were compared with our reference ranges. Therefore, ROC curves, sensitivity and specificity were obtained in consideration of the number of babies with favourable outcome whose CCTG parameters were falling between the 5th and the 95th percentile. The same evaluation was performed for unfavourable outcome babies.

\section{Results}

For all the traces, the median duration of the trace until criteria of reassurance were met (maximum $60 \mathrm{~min}$ ) was $32 \mathrm{~min}$ (Preboth 2000).

The median value for each parameter was calculated and their distribution through gestational age was elaborated. In Table 1 was reported the median value of CCTG paremeters first and before the 37th gestational week. FHR and ApEn showed a progressive decrease throughout pregnancy, while there is an increase of the values of STV, Delta and LTI. LF component and MF decreased, while HF increased. $\mathrm{LF} /(\mathrm{HF}+\mathrm{MF})$ ratio reduced. 
Table 1. Median value of cCTG parameters evaluated before and after 37 weeks of gestation.

\begin{tabular}{lcc}
\hline Parameters & Before the 37th week & After the 37th week \\
\hline FHR $(\mathrm{bpm})$ & $141 \pm 8.32$ & $138 \pm 8.01$ \\
STV $(\mathrm{ms})$ & $6.42 \pm 2.20$ & $7.12 \pm 2.42$ \\
LTI $(\mathrm{ms})$ & $21.86 \pm 6.36$ & $24.34 \pm 7.91$ \\
Delta (ms) & $41.38 \pm 11.99$ & $44.37 \pm 12.08$ \\
ApEn & $1.30 \pm 0.23$ & $1.26 \pm 0.25$ \\
LF $\left(\mathrm{ms}^{2}\right)$ & $83.78 \pm 5.10$ & $81.87 \pm 4.71$ \\
MF $\left(\mathrm{ms}^{2}\right)$ & $11.12 \pm 4.14$ & $12.35 \pm 3.27$ \\
HF $\left(\mathrm{ms}^{2}\right)$ & $5.59 \pm 3.22$ & $5.81 \pm 3.03$ \\
LF $/(\mathrm{HF}+\mathrm{MF})$ & $4.36 \pm 2.59$ & $4.06 \pm 2.14$
\end{tabular}

FHR: foetal heart rate; STV: short-term variability; LTI: long-term irregularity; ApEn: approximate entropy; LF: low frequency; MF: median frequency; HF: high frequency.

The Kruskal-Wallis test showed a statistically significant difference between gestational groups $(p<.05)$ for each parameter except for $\operatorname{HF}(p=.06)$ and $\mathrm{LF} / \mathrm{MF}+\mathrm{HF}$ ratio $(p=.75)$.

Reference normality values for each CCTG parameter according to gestational age for the 5th, 50thand the 95th percentile are reported (Table 2 ).

The Spearman's rank test (Figure 1) exhibited a decreasing FHR $(r=-.65, p=.02)$, ApEn $(r=-.098 ; p=.77)$, LF component $(r=-.87 ; p<.001)$ and $\mathrm{LF} /(\mathrm{HF}+\mathrm{MF})$ ratio $(r=-0.47$; $p=.11)$ with advancing gestational age. However, these tendencies were statistically significant only for FHR and LF component $(p<.05)$. In contrast, STV $(r=.59 ; p=.04)$, LTI $(r=.69$; $p=.01)$, Delta $(r=.67 ; p=.02)$, MF component $(r=.88$; $p<.001)$, and HF component $(r=.14 ; p=.65)$ showed to increase with advancing gestational age. These tendencies were statistically significant for STV, LTI, Delta and MF component $(p<.05)$. To examine the utility and performance of our reference ranges sensitivity and specificity were calculated. We obtained a sensitivity in detecting foetal distress of $90 \%$ and a specificity of $89 \%$ (confidence interval for sensitivity: 0.86-0.94; confidence interval for specificity: 0.81-0.93) (Tables 3 and 4). In Figure 2, ROC curve underlines the high diagnostic performance of our test with a value of AUC of 0,874(confidence interval: $0.834-0.941 ; p$ value $<.0001$ ).

\section{Discussion}

In the last years, computerised cardiotocography (cCTG) has established an important role in the medical management of pregnancy, especially in high-risk patients. The clinical practise requires a complex examination of pregnancy based on the combination of several foetal wellbeing methods. So, many authors tried to analyse the role of cCTG associated with the most established and well-known methods as Doppler ultrasonography, underling the high impact of this method in the clinical decision process. Siristadis had demonstrated that CTG, when combined with Doppler velocimetry, increases the clinicians' ability to accurately identify foetal hypoxia, and decreases the rate of caesarean section (Siristatidis et al. 2012). According to this growing role its necessary try to improve the method reducing its limitations.

In this investigation, a multiparametric analysis of cCTG signal through different linear and non-linear approaches both in time and frequency domain was proposed. Unlike previous studies (Arduini et al. 1993; Serra et al. 2009), this report offers a wider evaluation of CCTG parameters. Compared to Arduini's previous study (Arduini et al. 1993), we considered a larger sample for the analysis, starting from the 30th week of gestation and including the impact of spectral analysis. The importance of analysing the trend of cCTG parameters is related to the necessity of describing foetal neural response changes.

Our results agree with some previous studies (Dalton et al. 1983; Druzin et al. 1986; Serra et al. 2009), confirming that there is a significant change in foetal condition throughout gestation by the synergic action elicited by the Autonomic Nervous System (ANS) activity, through its sympathetic and parasympathetic branches (Pillai and James 1990; Guzman et al. 1996). The most evident modification consists of a significant decrease of baseline FHR during gestational age, which reflects the normal foetal neural maturity, because the progressive increase in the parasympathetic influence on foetal heart rate results in a gradual lowering of baseline rate.

STV is the most extensively studied parameter of computerised CTG, showing correlation with the presence of metabolic acidosis (Pincus 1991; Anceschi et al. 2003; Serra et al. 2008). In general, large variability reflects a healthy ANS and also normal activity of chemoreceptors, baroreceptors and cardiac responsiveness, while a decreased STV is associated with impending deterioration of foetal oxygen supply and finally intrauterine death. Overall, the main value of STV, LTI and Delta assessed by computerised 2CTG2 showed a statistically significant increase with gestational age. In a preterm period, reduction in foetal baseline variability may be due to incomplete development of ANS or may be associated to the characteristic tachycardia. ApEn has been introduced in based on the theory that human well-being is associated to an high level of irregularity of biomedical time series (e.g. heart rate, respiratory acidosis, and dynamics of hormone release) (Signorini et al. 2003; Xiaotian et al. 2005; Ferrario et al. 2006). We found that the mean value of ApEn showed a slight negative correlation with gestational age. A previous study indicated that extremely regular FHR pattern is not only associated with foetal hypoxaemia and acidosis, but also with respiratory and metabolic acidosis (Romano et al. 2006). This is the reason why the computation of ApEn could help in the detection of the early stages of foetal distress.

Spectral components of cCTG signal are strongly related to neural cardiovascular control system activity. They provide quantitative indicators of neural control of the sinoatrial node which can be useful to help the physician's evaluation of foetal wellbeing. Numerous experimental and clinical studies have consistently indicated the LF component as a marker of sympathetic modulation and HF as a marker of vagal modulation. $\mathrm{LF} /(\mathrm{HF}+\mathrm{MF})$ ratio is a synthetic index of sympathovagal balance (Struijk et al. 2001; Zhuravlev et al. 2002). During pregnancy, its reduction represents an imbalance of sympatho-vagal activity with predominance of parasympathetic tone. Spectral analysis also revealed a significant reduction of LF spectral component, because of the increase in the parasympathetic influence in cardiac regulation.

A complete evaluation of the clinical usability of cCTG should include the analysis of the potential adverse effects of this form of foetal assessment. For a long time CTG was 
Table 2. Reference ranges of the FHR, STV, LTI, Delta, ApEn, and spectral components throughout the third trimester of pregnancy, indicating 5th, 50th, 95th centiles.

\begin{tabular}{|c|c|c|c|}
\hline Gestational weeks & 5 th & 50th & 95th \\
\hline \multicolumn{4}{|l|}{ FHR (bpm) } \\
\hline 30 & 132.52 & 143.20 & 152.30 \\
\hline 31 & 128.15 & 140.48 & 153.92 \\
\hline 32 & 126.14 & 137.91 & 150.66 \\
\hline 33 & 130.62 & 140.01 & 155.21 \\
\hline 34 & 126.83 & 138.54 & 149.55 \\
\hline 35 & 128.58 & 142.93 & 157.40 \\
\hline 36 & 127.46 & 141.34 & 154.22 \\
\hline 37 & 126.82 & 139.07 & 152.23 \\
\hline 38 & 125.41 & 138.69 & 153.24 \\
\hline 39 & 127.13 & 137.93 & 149.26 \\
\hline 40 & 123.47 & 137.46 & 151.52 \\
\hline 41 & 122.13 & 136.93 & 152.28 \\
\hline 42 & 121.95 & 130.95 & 142.70 \\
\hline \multicolumn{4}{|l|}{ STV (ms) } \\
\hline 30 & 2.47 & 4.84 & 7.39 \\
\hline 31 & 3.23 & 6.19 & 9.62 \\
\hline 32 & 3.64 & 5.94 & 11.94 \\
\hline 33 & 3.08 & 5.75 & 9.09 \\
\hline 34 & 2.85 & 5.44 & 11.53 \\
\hline 35 & 3.32 & 6.64 & 9.89 \\
\hline 36 & 3.61 & 6.53 & 9.70 \\
\hline 37 & 3.31 & 6.79 & 10.58 \\
\hline 38 & 3.96 & 7.10 & 10.85 \\
\hline 39 & 3.31 & 6.72 & 12.20 \\
\hline 40 & 4.00 & 7.42 & 12.41 \\
\hline 41 & 3.51 & 6.83 & 11.54 \\
\hline 42 & 3.49 & 6.22 & 9.82 \\
\hline \multicolumn{4}{|l|}{ LTI (ms) } \\
\hline 30 & 11.46 & 17.55 & 27.79 \\
\hline 31 & 11.78 & 22.41 & 33.76 \\
\hline 32 & 13.38 & 21.96 & 36.56 \\
\hline 33 & 12.17 & 21.01 & 34.95 \\
\hline 34 & 10.56 & 20.34 & 36.42 \\
\hline 35 & 12.24 & 21.31 & 33.79 \\
\hline 36 & 14.02 & 21.78 & 31.28 \\
\hline 37 & 12.03 & 22.99 & 35.95 \\
\hline 38 & 12.53 & 24.91 & 42.87 \\
\hline 39 & 12.79 & 23.07 & 38.15 \\
\hline 40 & 14.19 & 23.82 & 35.49 \\
\hline 41 & 13.19 & 24.26 & 37.19 \\
\hline 42 & 11.09 & 23.82 & 37.07 \\
\hline \multicolumn{4}{|l|}{ Delta (ms) } \\
\hline 30 & 18.99 & 35.92 & 53.09 \\
\hline 31 & 22.77 & 42.89 & 69.22 \\
\hline 32 & 26.04 & 41.75 & 75.33 \\
\hline 33 & 23.93 & 40.05 & 61.49 \\
\hline 34 & 22.16 & 39.15 & 64.62 \\
\hline 35 & 19.86 & 41.76 & 63.78 \\
\hline 36 & 24.57 & 41.30 & 59.84 \\
\hline 37 & 22.04 & 42.67 & 63.84 \\
\hline 38 & 26.66 & 44.92 & 62.42 \\
\hline 39 & 24.31 & 43.87 & 68.99 \\
\hline 40 & 25.90 & 44.99 & 63.41 \\
\hline 41 & 24.52 & 44.57 & 63.82 \\
\hline 42 & 27.85 & 45.17 & 59.29 \\
\hline \multicolumn{4}{|l|}{ ApEn } \\
\hline 30 & 0.99 & 1.25 & 1.53 \\
\hline 31 & 0.99 & 1.30 & 1.62 \\
\hline 32 & 0.98 & 1.25 & 1.61 \\
\hline 33 & 1.00 & 1.29 & 1.58 \\
\hline 34 & 1.06 & 1.35 & 1.68 \\
\hline 35 & 0.94 & 1.36 & 1.80 \\
\hline 36 & 0.92 & 1.27 & 1.69 \\
\hline 37 & 0.97 & 1.28 & 1.65 \\
\hline 38 & 0.91 & 1.23 & 1.57 \\
\hline 39 & 0.84 & 1.19 & 1.59 \\
\hline 40 & 0.65 & 1.24 & 1.90 \\
\hline 41 & 0.98 & 1.33 & 1.66 \\
\hline 42 & 0.95 & 1.29 & 1.73 \\
\hline
\end{tabular}

Table 2. Continued

\begin{tabular}{cccc}
\hline Gestational weeks & 5 th & 50 th & 95 th \\
\hline LF $\left(\mathrm{ms}^{2}\right)$ & & & \\
30 & 77.43 & 84.13 & 90.94 \\
31 & 75.78 & 86.30 & 91.01 \\
32 & 75.82 & 85.90 & 90.06 \\
33 & 77.69 & 83.95 & 90.47 \\
34 & 74.82 & 84.62 & 90.27 \\
35 & 75.23 & 83.80 & 89.77 \\
36 & 73.74 & 83.74 & 88.66 \\
37 & 72.26 & 83.42 & 90.03 \\
38 & 71.96 & 82.81 & 89.03 \\
39 & 75.26 & 81.40 & 88.20 \\
40 & 74.15 & 81.53 & 88.37 \\
41 & 74.38 & 81.90 & 87.82 \\
42 & 75.02 & 80.95 & 88.78
\end{tabular}

$\mathrm{MF}\left(\mathrm{ms}^{2}\right)$

$\begin{array}{lrrr} & & \\ 30 & 6.46 & 9.80 & 14.92 \\ 31 & 4.09 & 8.70 & 15.27 \\ 32 & 6.58 & 9.66 & 16.92 \\ 33 & 7.63 & 10.04 & 16.40 \\ 34 & 9.77 & 15.56 \\ 35 & 5.90 & 10.23 & 17.03 \\ 36 & 6.03 & 11.14 & 17.45 \\ 37 & 7.39 & 11.82 & 17.99 \\ 38 & 7.63 & 11.49 & 17.50 \\ 39 & 7.30 & 12.40 & 16.82 \\ 40 & 7.32 & 13.03 & 19.16 \\ 41 & 8.00 & 12.80 & 18.45 \\ 42 & 6.65 & 10.72 & 19.47\end{array}$

$\mathrm{HF}\left(\mathrm{ms}^{2}\right)$

$\begin{array}{cccc}30 & 1.79 & 7.12 & 11.20 \\ 31 & 1.85 & 5.46 & 11.95 \\ 32 & 1.35 & 3.60 & 12.17 \\ 33 & 1.74 & 5.31 & 11.34 \\ 34 & 1.66 & 5.12 & 12.81 \\ 35 & 2.38 & 5.26 & 10.92 \\ 36 & 2.18 & 4.38 & 10.91 \\ 37 & 1.83 & 4.50 & 11.42 \\ 38 & 2.06 & 4.82 & 12.87 \\ 39 & 2.06 & 5.19 & 11.03 \\ 40 & 2.27 & 4.87 & 10.54 \\ 41 & 2.53 & 4.92 & 10.01 \\ 42 & 2.22 & 7.52 & 11.85\end{array}$

$\mathrm{LF} /(\mathrm{HF}+\mathrm{MF})$

\begin{tabular}{rrrr}
30 & 1.20 & 3.34 & 10.30 \\
31 & 1.24 & 3.96 & 9.91 \\
32 & 0.68 & 3.72 & 8.41 \\
33 & 0.59 & 3.21 & 9.22 \\
34 & 0.71 & 4.13 & 9.89 \\
35 & 0.90 & 4.40 & 8.81 \\
36 & 0.85 & 4.43 & 7.77 \\
37 & 1.02 & 4.12 & 9.34 \\
38 & 0.67 & 3.95 & 7.39 \\
39 & 1.15 & 4.25 & 7.34 \\
40 & 0.78 & 3.95 & 7.65 \\
41 & 1.41 & 3.48 & 6.87 \\
42 & 1.07 & 3.98 & 7.97 \\
\hline
\end{tabular}

FHR: foetal heart rate; STV: short-term variability; LTI: long-term irregularity; ApEn: approximate entropy; LF: low frequency; MF: median frequency; HF: high frequency.

Results are expressed as median (interquantile range) according to the gestational age expressed in weeks.

subjected to several criticisms due to the high number of false negative and false positive of the method with a false reassurance of foetal well-being for the mother and the health practitioner in the first case and unnecessary procedures or interventions for mother or foetus or newborn with increased use of healthcare resources, in the second case (Grivell et al. 2010). Thus, in this study we tried to elaborate a new system of classification of CCTG parameters for 

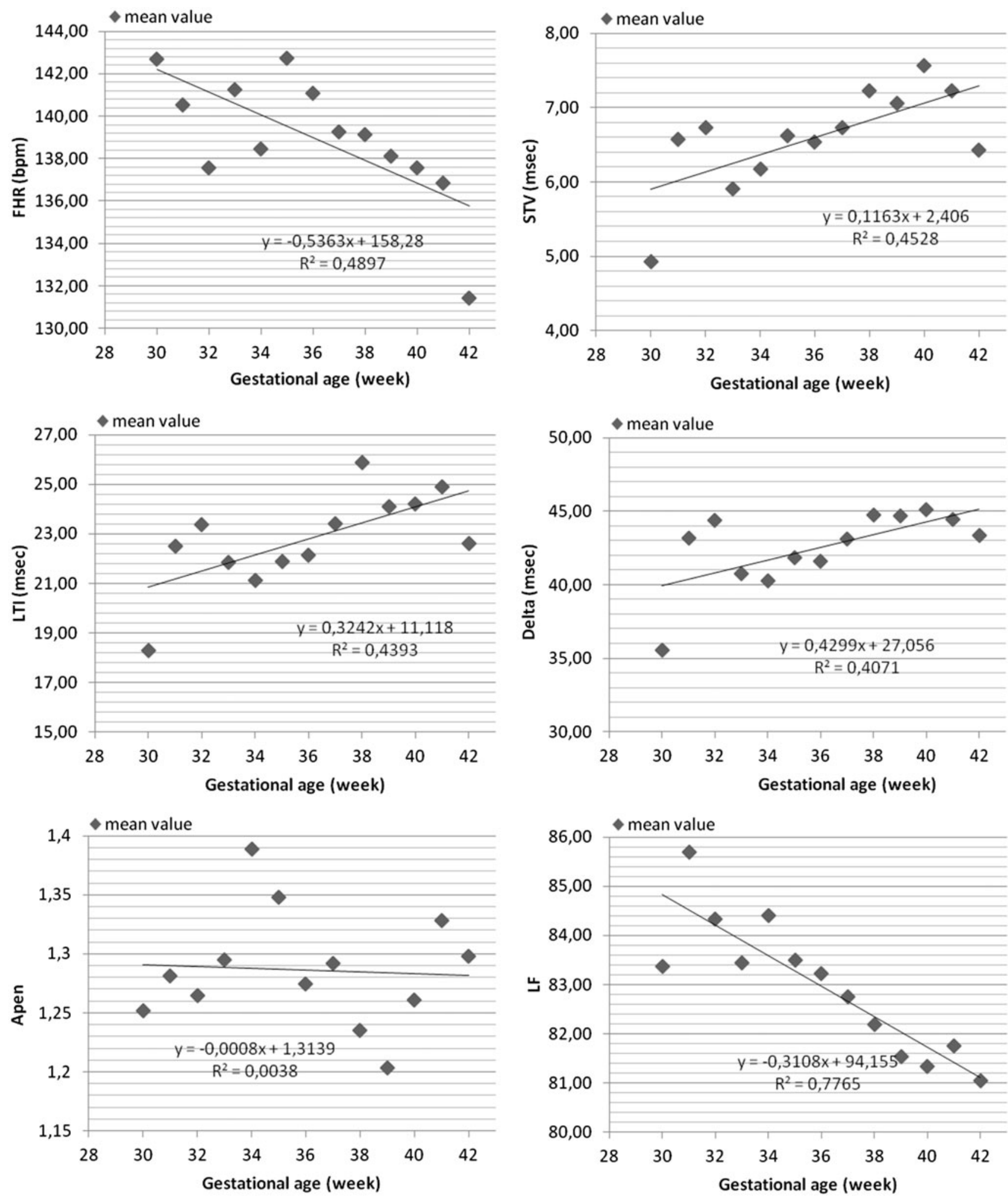

Figure 1. Relationship between CCTG parameters and gestational age evaluated with Spearman rank test.

improving the sensitivity and specificity of the method. The interpretation of foetal well-being requires a complete evaluation of pregnancy through clinical and instrumental analysis, but application of a test requires subsequent interpretation of the results according to what is defined or accepted as normal and abnormal. In the obstetric field, there is still no consensus on the best methodology for baseline estimation in computer analysis of CTG parameters. Many efforts were spent to find methods which permit to extract meaningful information, to classify or to describe the complex dynamical system which controls FHR variability, but with few attempts in evaluations how use that information. For these reasons we compared the reference values found with the foetal outcome, specificity and sensitivity were calculated considering 

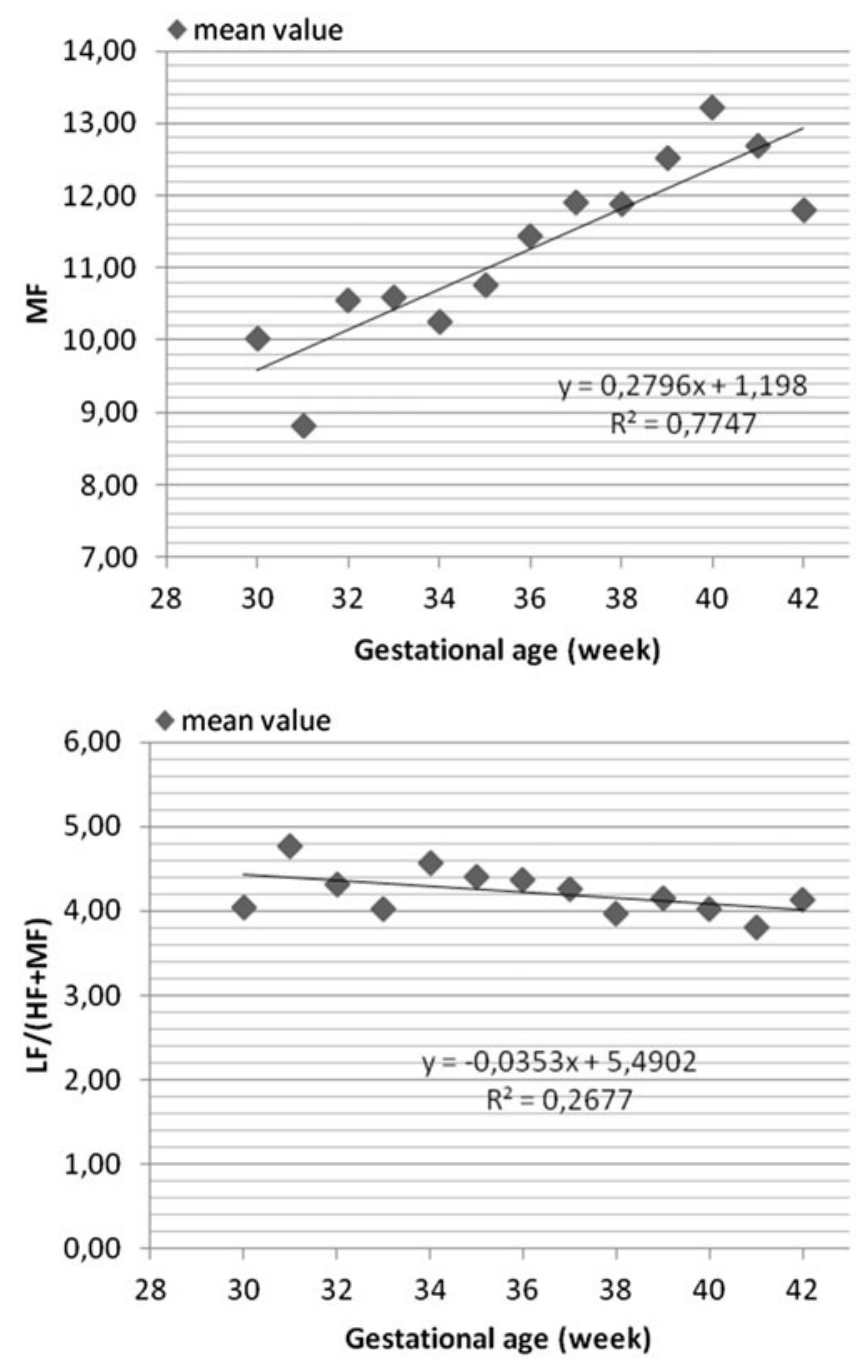

Figure 1. Continued.

Table 3. Relationship between neonatal outcome and antepartum cCTG traces.

\begin{tabular}{lcc}
\hline & $\begin{array}{c}\text { Unfavourable } \\
\text { neonatal outcome }\end{array}$ & $\begin{array}{c}\text { Favourable } \\
\text { neonatal outcome }\end{array}$ \\
\hline $\begin{array}{l}\text { cCTG parameters }<5 \text { th percentile } \\
\text { or }>95 \text { th percentile }\end{array}$ & $190(58 \%)$ & $13(3.9 \%)$ \\
$\begin{array}{l}\text { cCTG parameters between the 5th } \\
\text { and the 95th percentile }\end{array}$ & $20(6.1 \%)$ & $105(32 \%)$ \\
\hline
\end{tabular}

Table 4. Sensitivity and specificity.

\begin{tabular}{lcc}
\hline & Value & Confidence interval (95\%) \\
\hline Sensitivity & $90 \%$ & $0.86-0.94$ \\
Specificity & $89 \%$ & $0.81-0.93$ \\
False positive & 0.114 & $0.06-0.17$ \\
False negative & 0.093 & $0.06-0.13$ \\
VPP (positive predictive value) & 0.937 & $0.90-0.97$ \\
VPN (negative predictive value) & 0.835 & $0.77-0.90$ \\
Relative risk & 5.670 & $3.82-8.43$ \\
Odds ratio & 75.362 & $36.40-156.02$ \\
\hline
\end{tabular}

the last CCTG trace performed in the antepartum period (from the 37th week) for each patient before the onset of a spontaneous labour or an elective caesarean section. The $89 \%$ specificity suggested that an high percentage of foetuses (85\%) with a cCTG parameter between 5th and 95th
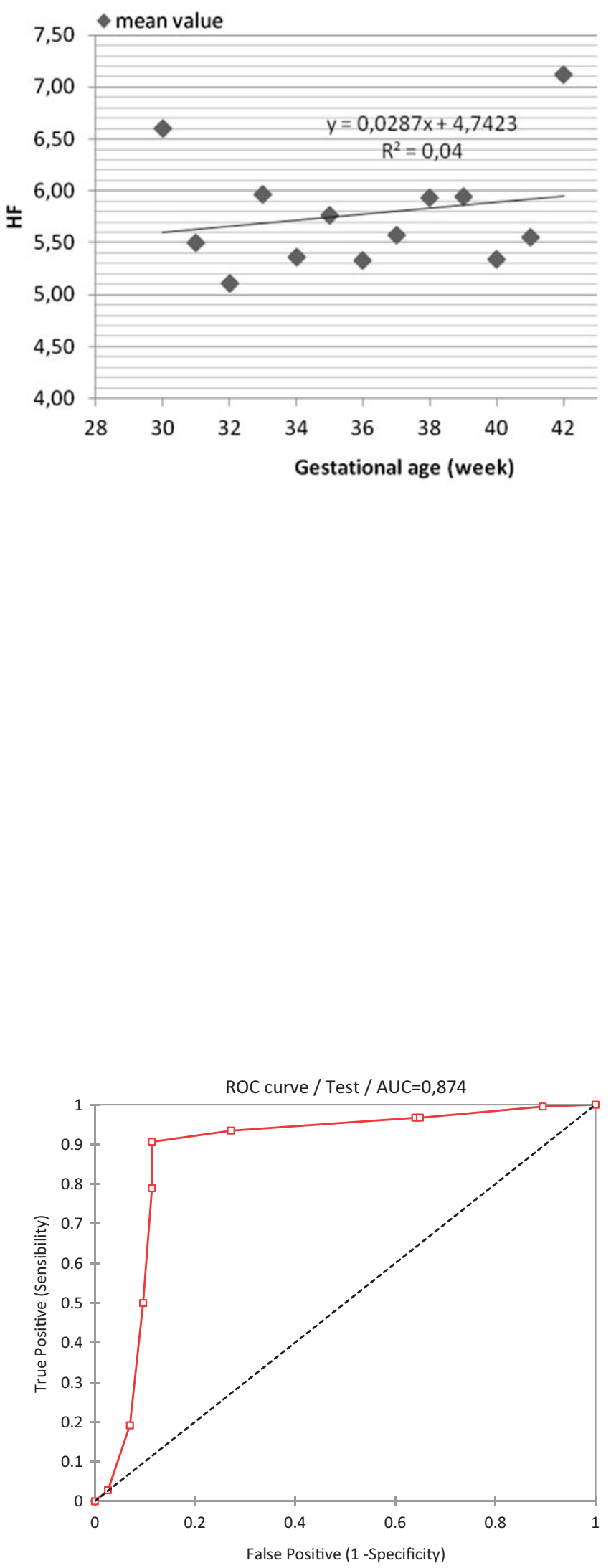

Figure 2. ROC curve underlines the high diagnostic performance of our test $(A \cup C=0,874)$.

percentile had a favourable outcome. Moreover, even if the specificity found is not significantly high, it represents a great result in comparison with traditional system (Dawes et al. 1991, 1996; Ayres-de-Campos et al. 2005; Costa et al. 2009). 
In fact, in our study, only $6,1 \%$ of the analysed babies had unfavourable outcome and antepartum trace between the 5th and the 95th percentile, while $3.9 \%$ of the babies with favourable outcome and CCTG parameters $<5$ th or $>95$ th percentile. These results suggest a possible improvement in pregnancy clinical management through the CCTG antepartum monitoring. A recent Cochrane del 2010 (Grivell et al. 2010) showed a significant reduction in perinatal mortality using CCTG versus traditional CTG. This study represents a further step in this direction and towards the prediction of foetal outcome based on non-invasive and standard technology.

\section{Strength and limitations of the study}

Even if our study was based on a large sample of patients, it was not a multicenter study; so our results could be perceived as of low clinical impact. However, our single-centre trial was based on a direct evaluation of all aspects of trial conduct, including data acquisition, quality control, data management, and data analysis. Moreover, the strength of the study is related to our evaluation of clinical application of the reference range calculated throughout the estimation of sensitivity and specificity. Finally, in our previous study (Annunziata et al. 2016), we showed that CCTG modifications during the different stages of labour reflected the physiologic increased activation of the autonomous nervous system. We believe that the use of computerised FHR analysis performed during labour it is possible to get more information from foetal cardiac signal, in comparison with the traditional tracing.

\section{Acknowledgements}

All the authors have fulfilled all the conditions required for authorship.

\section{Disclosure statement}

There are not conflicts of interest with the topic matter of this manuscript, as described in the instructions to authors.

\section{ORCID}

Salvatore Tagliaferri (iD http://orcid.org/0000-0002-8699-6544

\section{References}

American College of Obstetricians and Gynaecologists (ACOG). 1989. Intrapartum foetal heart rate monitoring. ACOG Technical Bulletin 132:1-6.

American College of Obstetricians and Gynaecologists (ACOG). 1995. Technical bulletin 207: foetal heart rate patterns: monitoring, interpretation, and management. International Journal of Gynecology and Obstetrics 51:65-74.

American College of Obstetricians and Gynaecologists (ACOG). 2005. Practise bulletin 70: intrapartum foetal heart rate monitoring. Obstetrics and Gynecology 106:1453-1460.

American College of Obstetricians and Gynaecologists (ACOG). 2009. Practise bulletin 106: intrapartum foetal heart rate monitoring: nomenclature, interpretation, and general management principles. Obstetrics and Gynecology 114:192-202.
Anceschi MM, Piazze JJ, Ruozi-Berretta A, Cosmi E, Cerekja A, Maranghi L, Cosmi EV. 2003. Validity of short term variation (STV) in detection of fetal acidemia. Journal of Perinatal Medicine 31:231-236.

Annunziata ML, Tagliaferri S, Esposito FG, Giuliano N, Mereghini F, Di Lieto A, et al. 2016. Computerized analysis of fetal heart rate variability signal during the stages of labor. Journal of Obstetrics and Gynaecology Research 42:258-265.

Arduini D, Rizzo G, Piana G, Bonalumi A, Brambilla P, Romanini C. 1993. Computerised analysis of foetal heart rate: I. description of the system (2CTG). Maternal Foetal Investment 3:159.

Ayres-de-Campos D, Costa-Santos C, Bernardes J, SisPorto Multicentre Validation Study Group. 2005. Prediction of neonatal state by computer analysis of foetal heart rate tracings: the antepartum arm of the SisPorto multicentre validation study. European Journal of Obstetrics and Gynecology and Reproductive Biology 118:52-60.

Bernardes J, Costa-Pereira A, Ayres-de-Campos D, Van Geijn HP, PereiraLeite L. 1997. Evaluation of interobserver agreement of cardiotocograms. International Journal of Gynaecology and Obstetrics 57:33-37.

Butt K, Lim K, Society of Obstetricians and Gynaecologists of Canada. 2014. Determination of gestational age by ultrasound. Journal of Obstetrics and Gynaecology Canada 36:171-183.

Costa A, Ayres-de-Campos D, Costa F, Santos C, Bernardes J. 2009. Prediction of neonatal acidemia by computer analysis of foetal heart rate and ST event signals. American Journal of Obstetrics and Gynecology 201:e1-e6.

Dalton KJ, Phill D, Dawes GS, Patrick JE. 1983. The autonomic nervous system and fetal heart rate variability. American Journal of Obstetrics and Gynecology 146:456-462.

Dawes GS, Moulden M, Redman CW. 1991. System 8000: computerized antenatal FHR analysis. Journal of Perinatal Medicine 19:47-51.

Dawes GS, Moulden M, Redman CWG. 1996. Improvements in computerized fetal heart rate analysis antepartum. Journal of Perinatal Medicine 24:25-36.

Druzin ML, Hutson JM, Edersheim TG. 1986. Relationship of baseline fetal heart rate to gestational age and fetal sex. American Journal of Obstetrics and Gynecology 154:1102-1103.

Ferrario M, Signorini MG, Magenes G, Cerutti S. 2006. Comparison of entropy-based regularity estimator: application to the foetal heart rate signal for the identification of foetal distress. IEEE Transactions on BioMedical Engineering 53:119-125.

Figueras F, Albela S, Bonino S, Palacio M, Barrau E, Hernandez S, et al. 2005. Visual analysis of antepartum foetal heart rate tracings: interand intra-observer agreement and impact of knowledge of neonatal outcome. Journal of Perinatal Medicine 33:241-245.

Gagnon R, Campbell MK, Humse C. 1993. A comparison between visual and computer analysis of antepartum foetal heart rate tracings. American Journal of Obstetrics and Gynecology 168:842-847.

Grivell RM, Alfirevic Z, Gyte GM, Devane D. 2010. Antenatal cardiotocography for foetal assessment. Cochrane Database of Systematic Reviews CD007863.

Guzman ER, Vintzileos AM, Martins M, Benito C, Houlihan C, Hanley M. 1996. The efficacy of individual computer heart rate indices in detencting acidemia at birth in growth-restricted foetuses. Obstetrics and Gynecology 87:969-974.

Hadlock FP, Harrist RB, Sharman RS, Deter RL, Park SK. 1985. Estimation of foetal weight with the use of head, body, and femur measurements-a prospective study. American Journal of Obstetrics and Gynecology 151:333-337.

Macones GA, Hankins GDV, Spong CY, Hauth J, Moore T. 2008. The 2008 national institute of child health and human development workshop report on electronic foetal monitoring: update on definitions, interpretation, and research guidelines. Obstetrics and Gynecology 112:661-666

Mantel R, van Geijn HP, Caron FJ, Swartjes JM, van Woerden EE, Jongsma HW. 1990. Computer analysis of antepartum fetal heart rate: 1. Baseline determination. International Journal of Biomedical Computing 25:261-272.

National Collaborating Centre for Women's and Children's Health, commissioned by the National Institute for Health and Clinical Excellence (NICE). 2007. Intrapartum care. London: RCOG Press.

National Institute of Child Health and Human Development research planning workshop (NICHHD). 1997. Electronic foetal heart rate 
monitoring: research guidelines for interpretation. American Journal of Obstetrics and Gynecology 177:1385-1390.

Nidhal S, Ali MMA, Zaidan AA, Zaidan BB, Najah H. 2011. Computerised algorithm for foetal heart rate baseline and baseline variability estimation based on distance between signal average and $\alpha$ value. International Journal of Pharmacology 7:228-237.

Pardey J, Moulden M, Redman CWG. 2002. A computer system for the numerical analysis of nonstress tests. American Journal of Obstetrics and Gynecology 186:1095-1103.

Park MI, Hwang JH, Cha KJ, Park YS, Koh SK. 2001. Computerized analysis of fetal heart rate parameters by gestational age. International Journal of Gynaecology and Obstetrics 74:157-164.

Pillai M, James D. 1990. The development of fetal heart rate patterns during normal pregnancy. Obstetrics and Gynecology 76:812-816.

Pincus SM. 1991. Approximate entropy as a measure of system complexity. Proceedings of National Academy of Sciences of the United States of America 88:2297-2301.

Pincus SM. 1995. Approximate entropy (ApEn) as a complexity measure. Chaos 5:110-117.

Preboth M. 2000 ACOG guidelines on antepartum foetal surveillance. American college of obstetricians and gynaecologists. American Family Physician 62:1184,1187-1188.

Romano M, Bracale M, Cesarelli M, Campanile M, Bifulco P, De Falco M, et al. 2006. Antepartum cardiotocography: a study of fetal reactivity in frequency domain. Computers in Biology and Medicine 36:619-633.

Royal College of Obstetricians and Gynaecologists (RCOG). 2001. The use of electronic foetal monitoring. Evidence-based clinical guideline, number 8. London: RCOG Press.

Serra V, Bellver J, Moulden M, Redman CW. 2009. Computerised analysis of normal foetal heart rate pattern throughout gestation. Ultrasound in Obstetrics and Gynecology 34:74-79.

Serra V, Moulden M, Bellver J, Redman CW. 2008. The value of the shortterm foetal heart rate variation for timing the delivery of growthretarded foetuses. BJOG 115:1101-1107.
Signorini MG, Magenes G, Cerutti S, Arduini D. 2003. Linear and nonlinear parameters for the analysis of fetal heart rate signal from cardiotocographic recordings. IEEE Transactions on Biomedical Engineering 50:365-374.

Siristatidis C, Kassanos D, Salamalekis G, Creatsa M, Chrelias C, Creatsas G. 2012. Cardiotocography alone versus cardiotocography plus Doppler evaluation of the foetal middle cerebral and umbilical artery for intrapartum foetal monitoring: a Greek prospective controlled trial. Journal of Maternal Foetal Neonatal Medicine 25:1183-1187.

Struijk PC, Ursem NT, Mathews J, Clark EB, Keller BB, Wladimiroff LW. 2001. Power spectrum analysis of heart rate and blood flow velocity variability measured in the umbilical and uterine arteries in early pregnancy: a comparative study. Ultrasound in Obstetrics and Gynecology 17:316-321.

Wróbel J, Horoba K, Pander T, Jeżewski J, Czabański R. 2013. Improving foetal heart rate signal interpretation by application of myriad filtering. Biocybernetics and Biomedical Engineering 33:211-221.

Xiaotian L, Daan Z, Shufeng Z. 2005. Approximate entropy of fetal heart rate variability as a predictor of fetal distress in women at term pregnancy. Acta Obstetricia et Gynecologica Scandinavica 84:837-843.

Yeh P, Emary K, Impey L. 2012. The relationship between umbilical cord arterial $\mathrm{pH}$ and serious adverse neonatal outcome: analysis of 51,519 consecutive validated samples. BJOG 119: 824-831.

Zhuravlev YE, Rassi D, Mishin AA, Emery SJ. 2002. Dynamic analysis of beat-to-beat fetal heart rate variability recorded by SQUID magnetometer: quantification of sympatho-vagal balance. Early Human Development 66:1-10.

Zuspan FP, Quilligan EJ, lams JD, van Geijn HP. 1979. Predictors of intrapartum fetal distress: the role of electronic fetal monitoring. Report of the national institute of child health and human development consensus development task force. American Journal of Obstetrics and Gynecology 135:287-291. 